\title{
Focal conformal fractionated radiotherapy vs. radiosurgery for lung cancer patients with limited brain metastases
}

\author{
Hung-Jen Chen ${ }^{1,2 \#}$, Chia-Hung Chen ${ }^{1,2 \#}$, Shuo-Chueh Chen ${ }^{1,2 \#}$, Hung-Lin Lin $^{3}$, Te-Chun Hsia ${ }^{1,2}$, \\ Chih-Yen Tu, ${ }^{1,4}$, Liang-Wen Hang ${ }^{1,2}$, Wen-Chien Cheng ${ }^{1,5}$, Chia-Chin Li $^{6}$, Chun-Ru Chien ${ }^{4,6,7}$ \\ ${ }^{1}$ Division of Pulmonary and Critical Care Medicine, Department of Internal Medicine, China Medical University Hospital, Taichung; ${ }^{2}$ Department \\ of Respiratory Therapy, College of Health Care, China Medical University, Taichung; ${ }^{3}$ Department of Neurosurgery, China Medical University \\ Hospital, Taichung; ${ }^{4}$ School of Medicine, College of Medicine, ${ }^{5}$ Department of Internal Medicine, Hyperbaric Oxygen Therapy Center, China \\ Medical University, Taichung; ${ }^{6}$ Department of Radiation Oncology, China Medical University Hospital, Taichung; ${ }^{7}$ Department of Radiation \\ Oncology, China Medical University Hsinchu Hospital, Hsinchu \\ Contributions: (I) Conception and design: All authors; (II) Administrative support: CR Chien; (III) Provision of study materials or patients: CR Chien; \\ (IV) Collection and assembly of data: CR Chien; (V) Data analysis and interpretation: CR Chien; (VI) Manuscript writing: All authors; (VII) Final \\ approval of manuscript: All authors. \\ \#These authors contributed equally to this work. \\ Correspondence to: Chun-Ru Chien, MD, PhD. School of Medicine, College of Medicine, China Medical University, No.91 Hsueh-Shih Road, North \\ District, Taichung. Email: d16181@gmail.com.
}

Background: For lung cancer (LC) patients with limited brain metastases (LBM), radiosurgery (RS) was the current preferred strategy. We aimed to report our experience regarding an alternative strategy (focal conformal fractionated radiotherapy, FCFRT) for these patients in this cohort study.

Methods: We identified LC patients with LBM treated with either FCFRT or RS within 2016-2019 without prior brain local treatment via in-house databases. The characteristics of patients, disease, treatment, and outcome were retrospectively obtained via chart review and peer review. The 1st day of FCFRT or RS was the index date. Overall survival (OS) was calculated from the index date to the last date of contact or death via the Kaplan-Meier method. Log-rank test was used in univariate analyses (UVA) whereas Cox regression method was used in the multivariate analyses (MVA). The incidence of local progression (LP) or distal brain metastases (DBM) was estimated by the competing risk approach with death as the competing risk.

Results: We identified 23 eligible patients. The median dose/fractionation for FCFRT was 36 Gy/10 fractions. The median dose for RS was 20 Gy. The Lung-molGPA prognostic groups' distribution for these two groups was not statistically different. After a median follow-up of 8 months (range, 1-38 months), the OS was not statistically different in UVA [P value 0.9]. The adjusted hazard ratio of death was 0.96 when FCFRT was compared to RS in MVA (95\% CI, 0.21-5.22). There was also no statistical significant difference in LP (P value 0.79 ) or DBM (P value 0.88 ).

Conclusions: For LC patients with LBM, the OS was not statistically different for definitive FCFRT or RS. There was also no statistical difference in LP or DBM. Further studies should be considered to clarify the indication of FCFRT.

Keywords: Brain metastases (BM); focal conformal fractionated radiotherapy (FCFRT); lung cancer (LC); radiosurgery (RS)

Submitted Dec 09, 2019. Accepted for publication Jul 28, 2020.

doi: 10.21037/apm-19-574

View this article at: http://dx.doi.org/10.21037/apm-19-574 


\section{Introduction}

Brain metastases (BM) occurred frequently in cancer patients, with lung cancer (LC) to be the most common primary site (1). For patients with limited BM (LBM), radiosurgery (RS) [such as gamma knife surgery (GKS)] or whole brain radiotherapy (WBRT) was available options according to current treatment guideline although the former was preferred (2). However, some other approaches such as focal fractionated regimens had been studied in the recent years (1).

In the past, the outcome of LC patients with LBM was still poor even treated with RS. In the two randomized controlled trials (RCTs) included in a systematic review published in 2018 (3), the median overall survival (OS) for LC patients with LBM treated with RS was 6.6 or 8.6 months $(4,5)$ respectively, with no benefit of adding WBRT.

Focal conformal fractionated radiotherapy (FCFRT) had been investigated for LBM. When we searched in PubMed using keywords "(((brain metastases) OR (brain metastasis)) AND (conformal) AND ((radiotherapy) OR (radiation therapy))) NOT (whole brain radiotherapy)" in Nov 2019, we identified two relevant studies providing information regarding definitive FCFRT for LBM. Ohtakara and Hoshi had reported median OS 12 months for 24 patients whereas 13.9 months was reported by Lockney et al. for 195 patients $(6,7)$. However, both studies were not limited to neither LC nor definitive setting. Furthermore, both studies were not compared with RS.

Due to the sparse literatures regarding FCFRT for LBM as mentioned above, especially for LC patients, we aimed to compare the clinical outcomes of LC patients with LBM treated with either definitive FCFRT or RS via retrospective review of patients treated at our institute. We present the following article in accordance with the STROBE Reporting Checklist (available at http://dx.doi. org/10.21037/apm-19-574).

\section{Methods}

\section{Study population}

Our study was a cohort study. We identified LC patients with LBM but without prior brain local treatment and treated with FCFRT or GKS (the main RS modality in our institute) within 2016-2019 by using in-house prospectively established databases. Our inclusion criteria included: (I) history of histological confirmation of LC; (II) LBM [defined as 1-4 metastases (8)] confirmed in image studies [magnetic resonance image (MRI) or computed tomography (CT) with/without position emission tomography (PET)]; (III) treated with FCFRT or GKS without prior brain local treatment. The characteristics of patients, disease, treatment, and outcome were retrospectively obtained via chart review and peer review. Our study was conducted in accordance with the Declaration of Helsinki (as revised in 2013) and approved by the Ethics Committee of our institute [CMUH106-REC3-119 (CR2)]. All participants gave informed consent before their treatments. We declared the patient's personal data have been secured.

\section{Treatment: FCFRT or GKS}

Patients in the FCFRT group were treated with 6- or 10- MV linear accelerators. Standard thermoplastic cast was used for immobilization then patients were simulated with CT in the treatment position. Gross target volume (GTV) was defined as the region of LBM in the simulation CT image, 2-5 $\mathrm{mm}$ margin with editing was added to form clinical target volume (CTV) for selected patients. We then added 3-5 mm margin for planning target volume (PTV) to be used in intensity-modulated radiotherapy (IMRT) planning. Image-guided radiotherapy (IGRT) was used in the setup of some patients by their preference [in need of out-ofpocket payment]. Patients in the GKS group were treated with standard stereotactic frame to obtain MRI for target delineation. GKS was delivered via the Leksell Gamma Knife C model (Elekta, Stockholm, Sweden).

\section{Statistical analyses}

The 1st day of FCFRT or GKS was the index date. OS was calculated from the index date to the last date of contact or death. Local progression (LP) [or distal brain metastases $(\mathrm{DBM})]$ was calculated from the index date to the $1^{\text {st }}$ date of LP (or DBM) confirmed by imaging work-up or the last date of imaging work-up without LP (or DBM). We excluded those cases with missing information. OS was estimated by Kaplan-Meier method and compared by logrank test in the univariate analysis (UVA). In order to adjust for potential differences in baseline prognostic factors, we used Cox regression method to adjust for the new and validated Lung-molGPA prognostic groups (LMGPG) in the multivariate analyses (MVA) (8-10). The incidence of LP or DBM was estimated by the competing risk approach with death as the competing risk (11). Chis-square test 
Table 1 Patient characteristics distribution for patients in overall survival analysis

\begin{tabular}{|c|c|c|c|}
\hline Characteristics & FCFRT & GKS & $P$ value \\
\hline \multicolumn{4}{|l|}{ Gender } \\
\hline Male & 8 & 5 & 1 \\
\hline Female & 6 & 4 & \\
\hline \multicolumn{4}{|l|}{ Histology } \\
\hline$A D$ & 11 & 9 & 0.25 \\
\hline Non-AD & 3 & 0 & \\
\hline \multicolumn{4}{|l|}{ PST } \\
\hline No & 4 & 5 & 0.38 \\
\hline Yes & 10 & 4 & \\
\hline \multicolumn{4}{|l|}{ CST } \\
\hline No & 2 & 1 & 1 \\
\hline Yes & 12 & 8 & \\
\hline \multicolumn{4}{|l|}{ Age } \\
\hline$\geq 70 \mathrm{y} / \mathrm{o}$ & 3 & 3 & 0.64 \\
\hline$<70$ y/o & 11 & 6 & \\
\hline \multicolumn{4}{|l|}{ KPS } \\
\hline$\leq 70$ & 2 & 0 & 0.58 \\
\hline 80 & 7 & 4 & \\
\hline $90-100$ & 5 & 5 & \\
\hline \multicolumn{4}{|l|}{ ECM } \\
\hline Without & 5 & 2 & 0.66 \\
\hline With & 9 & 7 & \\
\hline \multicolumn{4}{|l|}{ Gene $^{\dagger}$} \\
\hline Without & 6 & 1 & 0.18 \\
\hline With & 8 & 8 & \\
\hline \multicolumn{4}{|l|}{ LMGPG } \\
\hline $\mathrm{A} 2$ & 5 & 5 & 0.74 \\
\hline A3 & 6 & 4 & \\
\hline N2 & 2 & 0 & \\
\hline N3 & 1 & 0 & \\
\hline
\end{tabular}

${ }^{\dagger}$, positive vs. (negative or unknown) for epidermal growth factor receptor mutation or anaplastic lymphoma kinase fusion oncogene. AD, adenocarcinoma; CST, concurrent systemic therapy; ECM, extra-cranial metastasis; FCFRT, focal conformal fractionated radiotherapy; GKS, gamma knife surgery; GPA, graded prognostic assessment; KPS, Karnofsky Performance Score; LMGPG, lung-molGPA prognostic groups (A2: AD $2^{\text {nd }}$ group GPA score 1.5-2; A3: AD 3th group GPA score 2.5-3; N2: non- $A D 2^{\text {nd }}$ group GPA score 1.5-2; N3: non-AD 3th group GPA score 2.5-3); PST, prior systemic therapy; y/o, year old. or Fisher's exact test (when appropriate) were used for comparing categorical variables. Due to the small number of cases (see results), we did not perform additional subgroup or sensitivity analyses. Statistical analysis was performed using software R package "survival" \& "cmprsk".

\section{Results}

\section{Study population and treatment (Table 1)}

We identified 23 patients eligible for analyses (Figure 1). Most of them were male, adenocarcinoma, and had received prior or concurrent systemic therapy. The LMGPG distribution was not statistically different for FCFRT $v s$. GKS ( $\mathrm{P}$ value 0.74$)$. For those treated with FCFRT, the median radiotherapy dose and fraction were $36 \mathrm{~Gy}$ (range, 30-50 Gy) and 10 fractions (range, 5-22 months). A few of them used CTV $(n=6)$ or IGRT $(n=3)$. For those treated with GKS, the median marginal dose was 20 Gy [range, 15-22 Gy).

\section{Subsequent treatment and overall clinical outcomes}

All patients had received subsequent systemic therapy after FCFRT or GKS, whereas six patients had received additional local treatment during follow-up. At the time of analysis after a median follow-up of 8 months (range, 1-38 months), eight patients were dead. One patient treated with FCFRT was identified to have potential radiotherapy-related complication in need of inpatient care.

\section{OS}

The 1- and 2-year OS rate was not statistically different for FCFRT $v$ s. GKS in UVA (56\% and $56 \%$ (FCFRT) vs. $65 \%$ and $65 \%$ (GKS), $\mathrm{P}$ value 0.9$)$. The OS curve was shown in Figure 2. There was also no statistical difference in MVA with hazard ratio (HR) of death 0.96 when FCFRT was compared to GKS (95\% CI, 0.21-5.22).

\section{$L P$ and $D B M$}

The cumulative incidence of LP at 1 and 2 years were $31 \%$ and $31 \%$ for FCFRT (vs. 0 and $50 \%$ for GKS) without statistical significance (P value 0.79 ) as shown in Figure 3. The cumulative incidence of DBM at 1 and 2 years were $14 \%$ and $14 \%$ for FCFRT ( $v s .17 \%$ and $17 \%$ for GKS) without statistical significance (P value 0.88 ) as shown in Figure 4. 


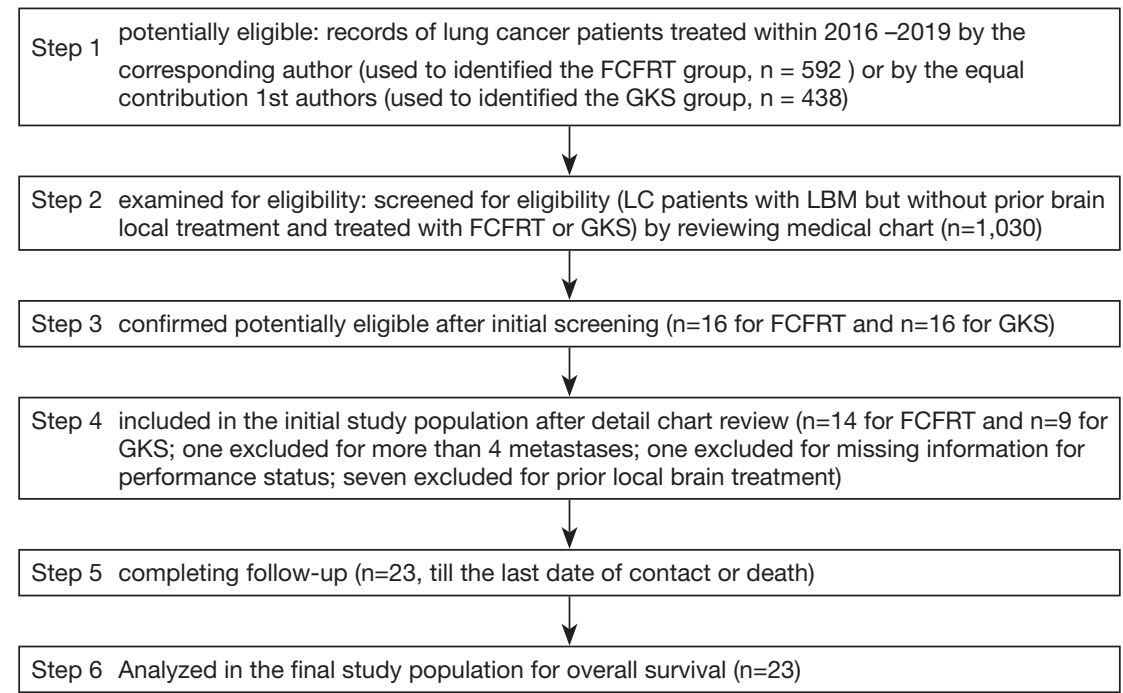

Figure 1 Study flow chart (STROBE format). FCFRT, Focal conformal fractionated radiotherapy; GKS, gamma knife surgery; LBM, limited brain metastasis; LC, lung cancer.

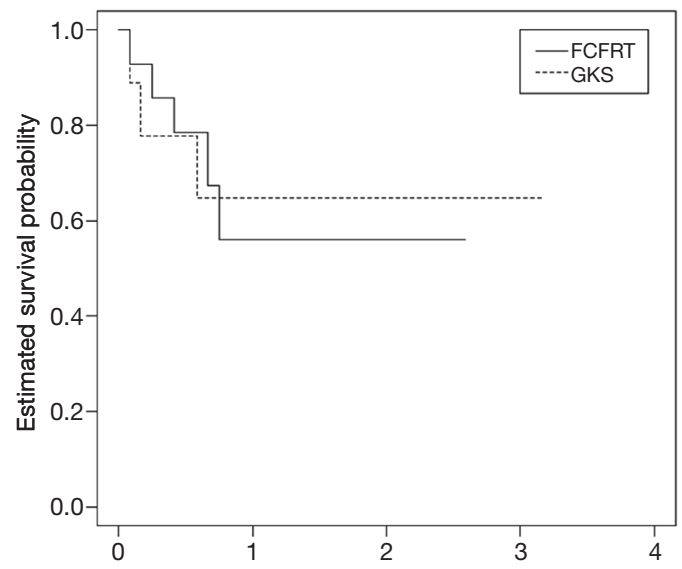

Figure 2 Kaplan-Meier overall survival curve (in years).

\section{Discussion}

For LC patients with LBM, we found that the OS was not statistically different for definitive FCFRT or RS using GKS. There was also no statistical difference in LP or DBM. This was the $1^{\text {st }}$ study to compare FCFRT $v s$. RS for LC patients with LBM to our knowledge.

Our results regarding FCFRT were slightly better to the literatures in which 1 year OS around 50\% and 2 year OS around $30 \%$ were reported $(6,7)$. Our results regarding RS were also slightly better to the literatures in which median OS around seven to nine months was reported $(4,5)$. Therefore, it is likely due to the advancement in

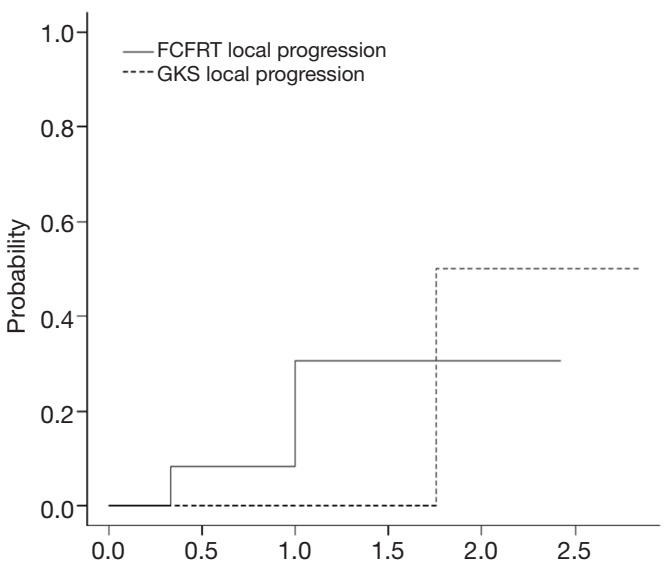

Figure 3 The cumulative incidence of local progression (in years).

the systemic therapy and overall care, which highlights the importance of studying contemporary patients with adjustment for modern prognostic score (8) as did in our study.

There were several limitations of our study. Obviously our sample size was relatively small, and the treatment selection [FCFRT vs. RS] was not randomized. Furthermore, cognitive or quality-of-life outcomes were not measured in our study. Therefore, the interpretation of our finding should be cautious, and studies of larger scale with additional outcomes, especially RCTs, should be considered to compare FCFRT $v s$. RS to clarify the indication of FCFRT for LC patients with LBM. However, 


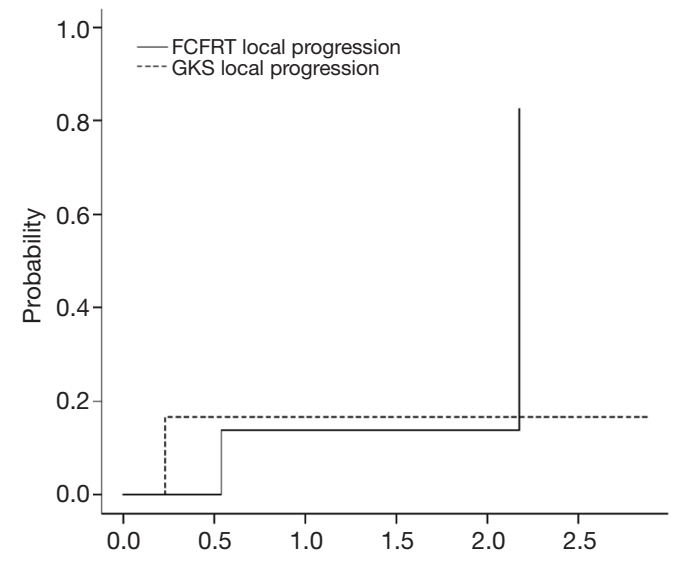

Figure 4 The cumulative incidence of distal brain metastases (in years).

when we searched clinical trial registry [https://clinicaltrials. gov/] in Dec 2019 using keywords (brain metastases I Lung Cancer Stage IV I radiosurgery I Phase 2, 3), we did not identify relevant studies. Therefore, our results provided a tentative evidence to consider FCFRT as an alternative for LC patients with LBM if RS was not favored.

\section{Conclusions}

For LC patients with LBM, the OS was not statistically different for definitive FCFRT or RS using GKS. There was also no statistical difference in LP or DBM. Further studies should be considered to clarify the indication of FCFRT.

\section{Acknowledgments}

This study was partially based on data from the China Medical University Hospital Cancer Registry. We thank Dr. Ji-An Liang (director of department of radiation oncology, China Medical University Hospital) for his administrative support in this study.

Funding: None.

\section{Footnote}

Reporting Checklist: The authors have completed the STROBE Reporting Checklist. Available at http://dx.doi. org/10.21037/apm-19-574

Data Sharing Statement: Available at http://dx.doi. org/10.21037/apm-19-574
Conflicts of Interest: All authors have completed the ICMJE uniform disclosure form (available at http://dx.doi. org/10.21037/apm-19-574). The authors have no conflicts of interest to declare.

Ethical Statement: The authors are accountable for all aspects of the work in ensuring that questions related to the accuracy or integrity of any part of the work are appropriately investigated and resolved. Our study was conducted in accordance with the Declaration of Helsinki (as revised in 2013) and approved by the Ethics Committee of our institute [CMUH106-REC3-119 (CR2)]. All participants gave informed consent before their treatments. We declared the patient's personal data have been secured.

Open Access Statement: This is an Open Access article distributed in accordance with the Creative Commons Attribution-NonCommercial-NoDerivs 4.0 International License (CC BY-NC-ND 4.0), which permits the noncommercial replication and distribution of the article with the strict proviso that no changes or edits are made and the original work is properly cited (including links to both the formal publication through the relevant DOI and the license). See: https://creativecommons.org/licenses/by-nc-nd/4.0/.

\section{References}

1. Tsao MN. Brain metastases: advances over the decades. Ann Palliat Med 2015;4:225-32.

2. National Comprehensive Cancer Network Guidelines for Central Nervous System Cancers, version 3.2019 [free registration required]. Accessed 17 Nov 2019. Available online: https://www.nccn.org/professionals/physician_gls/ $\mathrm{pdf} / \mathrm{cns}$.pdf

3. Qie S, Li Y, Shi HY, et al. Stereotactic radiosurgery (SRS) alone versus whole brain radiotherapy plus SRS in patients with 1 to 4 brain metastases from non-small cell lung cancer stratified by the graded prognostic assessment: A meta-analysis (PRISMA) of randomized control trials. Medicine (Baltimore) 2018;97:e11777.

4. Churilla TM, Ballman KV, Brown PD, et al. Stereotactic Radiosurgery With or Without Whole-Brain Radiation Therapy for Limited Brain Metastases: A Secondary Analysis of the North Central Cancer Treatment Group N0574 (Alliance) Randomized Controlled Trial. Int J Radiat Oncol Biol Phys 2017;99:1173-8.

5. Aoyama H, Tago M, Shirato H, et al. Stereotactic Radiosurgery With or Without Whole-Brain 
Radiotherapy for Brain Metastases: Secondary Analysis of the JROSG 99-1 Randomized Clinical Trial. JAMA Oncol 2015;1:457-64.

6. Ohtakara K, Hoshi H. Preliminary clinical outcomes of image-guided 3-dimensional conformal radiotherapy for limited brain metastases instead of stereotactic irradiation referral. Anticancer Res 2014;34:2997-3005.

7. Lockney NA, Wang DG, Gutin PH, et al. Clinical outcomes of patients with limited brain metastases treated with hypofractionated $(5 \times 6 \mathrm{~Gy})$ conformal radiotherapy. Radiother Oncol 2017;123:203-8.

8. Sperduto PW, Yang TJ, Beal K, et al. Estimating Survival in Patients With Lung Cancer and Brain Metastases: An

Cite this article as: Chen $\mathrm{HJ}$, Chen $\mathrm{CH}$, Chen $\mathrm{SC}$, Lin $\mathrm{HL}$, Hsia TC, Tu CY, Hang LW, Cheng WC, Li CC, Chien CR. Focal conformal fractionated radiotherapy vs. radiosurgery for lung cancer patients with limited brain metastases. Ann Palliat Med 2020;9(5):2600-2605. doi: 10.21037/apm-19-574
Update of the Graded Prognostic Assessment for Lung Cancer Using Molecular Markers (Lung-molGPA). JAMA Oncol 2017;3:827-31.

9. Nieder C, Hintz M, Oehlke O, et al. Validation of the graded prognostic assessment for lung cancer with brain metastases using molecular markers (lung-molGPA). Radiat Oncol 2017;12:107.

10. Hsu HS, Hu SL, Chien CR. A Prognostic Score for Brain Metastases of Non-small-cell Lung Cancer in the Era of Precision Medicine. Lung 2019;197:683.

11. Dutz A, Löck S. Competing risks in survival data analysis. Radiother Oncol 2019;130:185-9. 